\title{
Near-Earth Objects
}

Community White Paper to the Planetary Science Decadal Survey, 2023-2032

This White Paper was organized by the Small Bodies Assessment Group (SBAG)

Michael C. Nolan

Phone: +1 5206261978

University of Arizona

Email:mcn1@email.arizona.edu

Paul Abell, NASA Johnson Space Center

Erik Asphaug, University of Arizona

Christian Drouet d'Aubigny, University of Arizona

Tracy M. Becker, Southwest Research Institute

Marina Brozovic, Jet Propulsion Laboratory, California Institute of Technology

Michael W. Busch, SETI Institute

Richard P. Binzel, Massachusetts Institute of Technology

Steven R. Chesley, Jet Propulsion Laboratory, California Institute of Technology

R. Terik Daly, Johns Hopkins University Applied Physics Laboratory (JHU/APL)

Daniella DellaGiustina, University of Arizona

Maxime Devogele, Arecibo Observatory / University of Central Florida

Yanga R. Fernandez, University of Central Florida

Benjamin Greenhagen, Johns Hopkins Applied Physics Laboratory

Dylan C. Hickson, Arecibo Observatory / University of Central Florida

Ellen S. Howell, University of Arizona

Devanshu Jha, MVJCE, India

Hannah Kaplan, NASA Goddard Space Flight Center

Jian-Yang Li, Planetary Science Institute Ying Liao, National Central University Lucy F. Lim, NASA Goddard Space Flight Center
Sean M. Marshall, Arecibo Observatory / University of Central Florida Joseph Masiero, NASA JPL/Caltech

Patrick Michel, Université Côte d'Azur, Observatoire de la Côte d'Azur, CNRS, Laboratoire Lagrange, Nice, France

Shantanu P. Naidu, Jet Propulsion Laboratory, California Institute of Technology

Jessica Noviello, Arizona State University

Mark E. Perry, JHU/APL

Edgard G. Rivera-Valentín, Lunar and Planetary Institute (USRA)

Nalin Samarasinha, Planetary Science Institute

Carolina Rodríguez Sánchez-Vahamonde, Lunar and Planetary Institute, USRA

Alessondra Springmann, University of Arizona

Seiji Sugita, University of Tokyo

Timothy D. Swindle, University of Arizona

Patrick A. Taylor, Lunar and Planetary Institute, USRA

Flaviane C. F. Venditti, Arecibo Observatory / University of Central Florida

Ronald J. Vervack, Jr., Johns Hopkins Applied Physics Laboratory

Anne K. Virkki, Arecibo Observatory / University of Central Florida Luisa F. Zambrano-Marín, Arecibo Observatory / University of Central Florida 
Introduction

Asteroids and comets with perihelion distances $<1.3$ au are referred to as near-Earth objects (NEOs). NEOs are also, essentially by definition, the source of most meteorites that fall on the Earth. Because of encounters primarily with planets in the inner solar system, NEO orbits are fundamentally unstable with an average residence time of less than ten million years. NEOs are constantly replenished from the main asteroid belt and cometary reservoirs, primarily as a consequence of gravitational resonances with Jupiter and Saturn (see WP by Bottke et al.). In turn, objects in these resonance source regions are replenished by collisional diffusion of orbits and radiation forces (e.g., the Yarkovsky effect). There is compositional variation throughout the asteroid belt, with the inner region dominated by brighter silicate-rich objects and the outer region and Jupiter-family comets dominated by dark objects that are likely carbonaceous; however, there is substantial mixing across the belt. Because the NEO source regions span the main belt and Jupiter-family comets (though with widely variable efficiency), they are a diverse population. Although we have samples of NEOs via meteorite falls, the questions of how representative the meteorites are of the NEO population and how representative the NEO population is of the main-belt population are still open and will likely remain partially open even after the Hayabusa2 and OSIRIS-REx samples are analyzed (see WP by McAdam et al.).

\section{$2 \quad$ Why study of NEOs is a compelling undertaking}

NEOs are important for three main scientific reasons: as samples of their parent bodies, as potential hazards to the Earth, and as the next step in human exploration.

As samples of main-belt asteroids and comets, NEOs provide clues to the nature of the early solar system. These objects are part of the surviving population of planetesimals from the very earliest stages of solar system formation. These bodies thus provide the primary constraints on the processes and conditions that were present during the formation epoch and can be used to test current models and theories describing the late solar nebula, the early solar system, and subsequent planetary accretion (see WP by Davidsson et al.). From detailed knowledge of NEO compositions, probable starting materials and thermal histories of their asteroid and comet parent bodies can be inferred. Further, because meteorites and interplanetary dust particles (IDPs) represent the primary evidence for the environments and mechanisms of the formation of the solar system, if specific meteorite and IDP groups can be compositionally associated with NEOs, and the NEOs tied to main-belt asteroids and comets, the interrelations between all these objects can be tested, providing a dynamical context for the in-depth laboratory studies of meteorites and IDPs.

As potential hazards, NEOs have the potential to significantly and adversely affect life on Earth. It is now generally accepted that a $\sim 10 \mathrm{~km}$ extraterrestrial body hit the Earth causing the transition between the Cretaceous and Paleogene periods (K/Pg) and caused a massive extinction of the existing flora and fauna. NEOs will impact the Earth in the future. Therefore, NEOs have been the focus of ground-based surveys in recent years in order to discover and, to a lesser degree, characterize these bodies. Detailed investigations of these objects would therefore provide an extremely important scientific resource, as well as an invaluable data set on NEO physical characteristics (e.g., physical strength, density, etc., as well as orbits) that would be of vital importance in the development of possible mitigation strategies for any hazardous NEO. Mitigation strategies depend in detail on the physical state of the impactor. Density, degree of 
fragmentation, and presence or absence of satellites are all critical information for planning any potential mitigation strategy. This topic is examined in detail in the WP by Mainzer et al.

The next step in the human exploration and utilization of space will be highly dependent on extracting materials (primarily water and minerals) from in-situ sources. NEOs are our nearest neighbors in the solar system after the Moon, and are even nearer in $\Delta \mathrm{V}$. Thus, they are potentially the most cost-efficient sources for providing life support and propulsion and for building structures in space. It is highly probable that the success and viability of human expansion into space beyond low-Earth orbit will depend on the ability to take advantage of these potential NEO resources. Therefore, a detailed physical and compositional assessment of the population will be required during the next decade before human missions are sent to these objects. This topic is discussed in detail in the WP by Adamo et al.

NEOs share these motivations and scientific questions with their immediate progenitors, main-belt asteroids (see WP by McAdam et al.) and comets and are particularly important for the simple reason that they are some of the easiest objects to reach in the solar system, both by spacecraft and with ground-based characterization. These objects are accessible owing to their relatively low $\Delta \mathrm{V}$ (5 to $6 \mathrm{~km} / \mathrm{s}$ ) and are thus prime targets for possible future science and sample return missions. For the next decade of planetary science research, we need to identify and characterize targets within the NEO population for future spacecraft missions, develop technologies required to interact with these objects, design robotic spacecraft to explore/sample NEOs, and determine the potential for human exploration. Because the NEOs are intermediate objects between the meteorite samples we study on the Earth and the planetesimals that formed at the start of solar system history, they are important objects for teaching us how the original nature and variety of rocky planetesimals is or is not represented in the meteorite collection.

\section{Major Scientific Questions}

Although much progress has been made in recent years towards answering the following top-level science questions and their more specific sub-questions, our current understanding of these issues is far from complete. These areas demand further investigation through a wellbalanced program combining ground-based and space-based NEO observations, targeted spacecraft missions, laboratory research, fireball detection to aid meteorite recoveries, and data analysis activities. These efforts should focus on addressing the following key questions identified by the Small Bodies Assessment Group (SBAG 2020):

\subsection{What is the distribution of NEOs today, and how has material migrated from where it initially formed?}

\subsubsection{What is the Compositional Distribution of NEOs?}

Answering this question requires understanding and compensating for discovery selection effects, understanding how to relate remote observations to specific mineralogies and how surface mineralogies relate to bulk composition. It requires understanding the nature of "space weathering” on NEOs and how it varies with composition, presence of regolith, and object size. The answers provide insight into their geological diversity, the relationship between NEOs and collected meteorite and IDP samples (which in turn provide more detailed information about NEO formation and evolution) and the existence and abundance of key resources on NEOs (i.e., water, minerals, metals, organic molecules, etc.).

\subsubsection{What are the Specific NEO Source Regions and Sinks?}

Dynamical mechanisms and their efficiency for transferring objects from different regions in the main asteroid belt and elsewhere must be understood as well as the composition 
and physical characteristics of the source region populations. In this sense, NEO monitoring programs can provide accurate orbital information for meter-scale impactors such as 2008 TC3, which allow better identification of the dynamic resonances delivering meteorites from the main belt, and also identify NEOs producing meteorites. Detailed modeling of dynamical evolution is required to understand NEO interactions with the inner planets, including Earth, resulting in scattering, disruption or accretion. These mechanisms also provide insights into the role of NEOs in the delivery of water and prebiotic materials to the Earth. A thorough investigation of the NEO population that bears all of these diverse goals in mind will yield the broadest results regarding the scientific, hazard, mitigation, and resource potential of NEOs.

3.1.3 What are the physical properties and key processes on NEOs and how are they modified over time?

The important physical properties of NEOs include their size-frequency and spin-state distributions, which provide constraints on their collisional evolution and other processes affecting these distributions. Interior structure can be probed directly by using dedicated instruments on board spacecraft (see WP by Haynes et al.) or potentially indirectly via a knowledge of spin states coupled with size and shape, which can provide insights into density, strength, and interior structure. Understanding NEO evolution requires an understanding of impact processes, radiation processes (e.g., YORP) and their timescales. Recently, the view of most NEOs has undergone some major changes consistent with a) the now widely accepted rubble-pile model for the structure of NEOs larger than $\sim 100 \mathrm{~m}$ and $\mathrm{b}$ ) the implications of spinstate alteration) through YORP and similar effects. YORP-induced spin-up can lead to "landsliding" or redistribution of material to produce surface features. By observing these features, we can constrain the material properties of the rubble pile material such as coefficients of friction. Particles have been observed to be in temporary orbit around Bennu, resulting in some material being lost, but most falling back to the surface. We do not yet know how common this phenomenon is for NEOs in general, or what the production mechanism is for this unexpected process. Binary asteroids represent an in-situ laboratory for studying these processes, where they are actively modifying the system on human-accessible timescales. They are likely to shed mass during their evolution and may contribute significantly to the meteorite population. The large fraction of NEO binaries suggests that they represent a common part of an asteroid's "life cycle."

\subsection{How can NEOs be used as resources?}

The dynamically closest objects are the most accessible for exploration and/or exploitation and possess the highest Earth-impact probabilities. Bulk materials from rubble-pile asteroids could provide building materials and shielding, and the availability of water (for drinking, oxygen, and propellant) is a critical factor in extending the human presence in space. There is considerable evidence that many C-class (and some M-class) asteroids contain significant amounts of water in the form of hydrated minerals. Even S-type asteroids show some evidence of hydrated surface material. Identification of NEOs most likely to contain such minerals is thus a necessary precursor to developing a long-term manned presence in space. Final assessment of the accessibility and quantitative abundance of these resources on candidate asteroids will require spacecraft reconnaissance. However, confirming the existence of water and identification of NEOs that contain it can be met using existing techniques and instruments. Ground-based studies provide information to address this issue for numerous main-belt asteroids and for many NEOs, although a small fraction of the known population. 


\section{$4 \quad$ Required Research and Research Facilities}

\subsection{Discovery and Characterization}

These activities are the foundation of any scientific investigation of NEOs. A general survey of the dynamical and physical characteristics of the NEO population as a whole is a necessary first step towards achieving a more sophisticated understanding of these objects. Once enough data have been acquired from a representative sample of the NEO population, more educated decisions concerning spacecraft target selection can be made. To date (and for the foreseeable future) most mission targets have been selected primarily on accessibility: required $\Delta \mathrm{V}$ and cruise duration are fundamental to target selection. Only by greatly expanding the number of well-characterized asteroids can we expand the number of mission opportunities and explore options for multiple NEO targets for a single mission. Maintaining these programs decreases overall mission risk and simplifies spacecraft designs, which corresponds to a reduction in mission cost while still achieving the desired scientific and exploration objectives. The close approach of asteroid Apophis on April 13, 2029 presents a unique opportunity to study a potentially hazardous NEO in great detail. See WPs by Binzel et al. and Cheng et al.

\subsubsection{Asteroid Discovery Programs}

We estimate that there are 20,000-30,000 NEOs larger than $140 \mathrm{~m}$ diameter of which 9177 are known (July 4, 2020; http://neo.jpl.nasa.gov/stats/). This number is increasing by several hundred each year, primarily as the result of current NEO search programs. It is vital that these programs continue until next-generation surveys such as LSST and NEOSM reach operational maturity and demonstrate that they are more efficient in the long term and can provide rapid dissemination of objects for characterization. Even after the discovery goals are met, ground-based discovery programs will allow us to detect terminal-discovery impactors like 2008 TC3, which are of enormous scientific and geopolitical importance. 2008 TC3 is the only ureilite parent body we have been able to characterize. Because of its discovery in a routine survey - it was not a fluke discovery — it was well characterized by numerous telescopes before impact and provides important ground truth for linking observable properties before (NEOs) and after atmospheric entry (meteorites).

\subsubsection{Telescopic Characterization.}

A robust and ongoing program to obtain physical observations to determine rotation rates, pole directions, sizes, shapes, and compositions of NEOs is needed. This can most efficiently be done using ground-based facilities, which can observe orders of magnitude more objects than spacecraft reconnaissance at orders of magnitude lower cost. In particular, groundbased radar observations are a powerful technique for obtaining such data on close-approaching NEOs and providing a unique capability to image them (see WP by Taylor et al.). These images reveal geophysical features and the variety of physical processes that alter them (see WP by Virkki et al.). In order to meet these requirements, we need to: expand current programs; provide instrumentation to cover the full 0.4 to 5 micron spectral range; and make available larger (5-6m) telescopes instrumented for NEO observations to enable study of smaller NEOs, improve albedo information, (e.g., via direct imaging, polarization observations, or multi-wavelength radiometric data) and better constrain mineralogical identifications. The WPs by Abell et al. and Barbee et al. have recommendations specific to planetary defense. Extending the spectral coverage below 0.15 microns via space-based telescopes would enable space weathering measurements and sensitive searches for the presence of water and iron oxides on NEO surfaces. 


\subsubsection{Rendezvous and in situ Physical Characterization}

An ongoing program of robotic missions to rendezvous and characterize a number of NEOs is needed to accomplish the overall NEO science goals for the next decade. Because the NEO population is a diverse and complex population of bodies, the overall strategy should be to visit a number of objects of differing compositions and internal structures, as discussed in a WP by Jacobson and Bose. Binary NEOs are particularly interesting and present additional challenges and unique opportunities, e.g., Janus. Such a program should be optimized based on a common spacecraft design for this initial reconnaissance, and would ideally deploy spacecraft to, or visit, multiple NEOs with a single launch vehicle to reduce mission cost and program risk. In situ physical characterization missions would help to discover interesting prospects among the NEO population for further investigation, both via robotic and crewed spacecraft, and identify possible future sample return mission targets.

\subsubsection{Sample Return}

Despite considerable progress with determining composition by remote methods, many puzzles remain that are only tractable by sample return. The upcoming sample returns from Bennu and Ryugu will address primitive objects, but other common types of asteroids (and meteorites) are not well sampled and are ripe for sample return. For example, clinopyroxene can be detected spectroscopically and is generally assumed to indicate melts or partial melts, but it is equally diagnostic of unequilibrated ordinary chondrites, the most primitive (unmetamorphosed) ordinary chondrites. Another example is chronology, which is essential for understanding the history of solar system materials, but there is as yet no demonstrated means to do this remotely or by in situ methods. A third example is physical studies to yield density, porosity, etc.

Meteorites are a highly biased (selection during creation, solar system transport, and atmospheric passage) subset of the asteroid population, representing the toughest material. They are therefore probably not completely representative of the material observed by asteroid astronomers. Sample return can provide unconsolidated regolith, with its wealth of information about the space environment and the local and distant geology of the asteroid (viz. lunar regolith studies).

\subsection{Technology Needs}

New research and technology development programs are needed in order to achieve the goals and objectives of NEO research for the future decade in the following areas:

- Remote sensing and characterization

- $\quad$ Spacecraft systems (e.g., guidance, navigation, propulsion, autonomy, miniaturization)

- Surface mobility and sample collection

- In situ resource utilization

- Telecommunications for "near-deep-space” smallsat missions

One of the biggest issues in NEO research is the quality and quantity of the data for astrometry and characterization. In order to maintain pace with the discovery programs, ground-based characterization efforts should be expanded Because telescope instrumentation requirements for NEOs (and planetary surfaces in general) differ from those for more classical "astronomical” targets, new instrumentation, designed for planetary observations, should be developed. They should cover the entire 0.4-5.0 micron spectral range at individual telescopes so that spectra can be obtained in a single night to better constrain NEO compositions. Low spectral resolution optimized for faint NEOs would be best suited to asteroid observations. NEO observations require very accurate time information and rapid non-sidereal tracking. More observing time on larger (5-6m) telescopes will be required to enable the study of smaller NEOs. 
In addition to the above needs for augmentation of ground-based observing facilities and improvement of their operation and coordination, specific small-body reconnaissance and/or sample return missions require further development of spacecraft technologies. Few of these development programs are unique to the study of NEOs, but the needs for navigation and proximity operations in the vicinity of NEO mission targets are complex owing to their small sizes, possible satellites, and irregular gravity environments. Requirements include propulsion and power, telecommunication, remote sensing (e.g. radar tomography, seismic studies, etc.), surface sampling, guidance navigation and control, and autonomy technologies. In addition, spacecraft deployed assets (landers, rovers/hoppers, penetrators, etc.) interacting with a NEO's surface under microgravity conditions are also needed to maximize the science potential and permit resource utilization and manned exploration.

\section{Major Mission Priorities over the Decade 2023-2032 \\ 5.1 Flagship missions \\ No flagship missions to NEOs are proposed over the decade 2023-2032. \\ 5.2 New Frontiers Class mission(s) \\ A New Frontiers-class grand-tour mission should be developed.}

A Grand Tour mission to rendezvous with and orbit a number of NEOs of a variety of classes, in particular objects that may not be represented in the meteorite population. Only a spacecraft mission is capable of elucidating the distinctions between the various compositional classes and providing the in-depth observations needed to understand the details of formation of different physical types. Such a spacecraft could have several (perhaps three or four) penetrators, microlanders, or similar low-cost easily deployed surface exploration modules. Remote sensing instruments (laser altimeters, vis/IR spectrometers, gamma and neutron spectrometers, and ground penetrating radars) provide details of the physical and chemical evolution of these bodies.

\subsection{Discovery and Smallsat Science Goals}

\subsubsection{Reconnaissance of the population of NEOs}

Asteroid rendezvous missions (which can include flybys) could visit multiple NEOs, using remote sensing to connect their taxonomic types with meteorite classes, and to examine their small-scale structure inaccessible to ground-based imaging, and their interiors through the use of radar (see WP by M. Haynes). Owing to the low required $\Delta \mathrm{V}$ to reach many NEOs, and benefiting from advances in technology and operations heritage, future Discovery-class missions can also meet ambitious science objectives requiring surface operations, ballistic interactions, and sample return. Such missions necessarily make assumptions about the physical nature of the surface, so reliance on low-cost autonomous deployables can mitigate risk. Smallsats now have proven capabilities in deep space, and miniaturized instrumentation makes NEAR-type missions possible at much lower cost, or a fleet of such missions under Discovery. Semi-autonomous operations would allow these missions to take data over the course of an orbit and return it to Earth during conjunctions, the biggest hurdles being propulsion and communications.

\subsubsection{NEO Search from a space-based platform.}

A space-based discovery platform has clear performance advantages over the same-size ground-based telescope owing to the lack of atmospheric absorption (particularly in the IR), weather, continuous operation, and diffraction-limited optical performance. Space-based platforms do have more limited lifetimes, higher costs, and more restricted operating modes than do ground-based systems, as they require that the survey be properly scoped and defined prior to 
launch. The Near-Earth Object Surveillance Mission (NEOSM) should be supported (see WPs by Mainzer et al. and Barbee et al. on NEOSM).

\section{$6 \quad$ Balancing Priorities}

Increased support for ground-based observations of NEOs at 5-6m telescopes and for analysis of existing data from recent and ongoing missions will lead to a better foundation for future NEO studies and place their science results in proper context. In addition, in order to provide good foundations for the selection of future NEO missions, and the subsequent interpretation of their data, efforts should be made to include a mix of survey operations, spectral and physical characterization studies, radar observations, laboratory analyses, technology development, and theoretical modeling in well-supported and consistently funded NASA research and data analysis programs. These budgets should be stable and keep up with inflation.

Ground-based and space-based observations can discover and characterize a significant fraction of the estimated NEO population. These assets can also identify the future mission candidates for investigation, whereas in situ spacecraft reconnaissance will provide answers to the detailed questions that cannot be answered via remote sensing methods.

\section{Recommendations}

An NEO research program should incorporate elements of both remote sensing and spacecraft in situ physical characterization. For the next decade of planetary science research, we need to identify and characterize targets within the NEO population for future spacecraft missions, develop technologies required to interact with these objects, design robotic spacecraft to explore/sample NEOs, and explore the potential for human exploration. To do so, we recommend:

- Support for the Near Earth Object Survey Mission (NEOSM).

- Maintenance of ground-based asteroid discovery programs until the next-generation of surveys (Rubin LSST and NEOSM) are operationally mature, then maintain ground-based discovery infrastructure to concentrate on near-term potential impactors and follow-up.

- Funding the development of NEO-specific instrumentation, which has different requirements than astrophysics instrumentation (low resolution, wide wavelength range, accurate timing).

- Funding full-time equivalent access to a 5-6m telescope instrumented for NEO observations.

- Maintenance of a mix of survey operations, spectral and physical characterization studies, radar observations, laboratory analyses, technology development, and theoretical modeling in well-supported and consistently funded NASA research and data analysis programs.

- Development of a New Frontiers-class NEO grand-tour mission.

- Development of Discovery and Smallsat missions to visit a number of objects of differing compositions and internal structures.

- Pursuit of technology development for spacecraft proximity operations and Smallsat propulsion and communications.

- Development of a plan to study the Apophis encounter with Earth in 2029.

\section{Reference}

SBAG (2020), Goals and Objectives for the Exploration and Investigation of the Solar System’s Small Bodies. ver. 2.0.2020, 44 p., at http://www.lpi.usra.edu/sbag/goals/ 